http://dx.doi.org/10.21611/qirt.1992.026

\title{
Thermomechanical evaluation of polypropylene fracture resistance
}

\author{
by G. MEDRI (*) and R. RICCI (**)
}

$\left(^{*}\right)$ Istituto di Ingegneria Meccanica, Università di Salerno, Italy.

$\left(^{\star *}\right)$ Dipartimento di Energetica, Università di Ancona, Italy.

\begin{abstract}
The results of thermomechanical analyses performed on polypropylene specimens during fracture tests are presented. A thermodynamic approach to the fracture mechanics of dissipative materials is discussed.
\end{abstract}

\section{Introduction}

Present fracture mechanics analyses of dissipative materials rely on basically elastic energetic approaches, taking into account the anelastic component of material behaviour via conventional experimental methodics or more-or-less empirical updating.

The general stability criterion for cracked bodies is fracture is unstable whenever the total stored elastic energy decrease per unit crack growth is greater than a critical quantity. This critical quantity may named fracture (or tearing) energy.

In the case of viscoelastic bodies, the evaluation of the elastic energy rate linked to time-depending loads and fracture length in non-isothermal conditions (i.e. in actual conditions) is very difficult, even if not impossible in principle. So, the evaluation of the fracture toughness of polymeric structures is limited by the difficult determination of the critical parameter and by the lengthy and expensive test campaigns necessary.

The authors designed and built a testing device coupled with IR camera which allows experimental studies of thermal fields in the neighbour of cracks inserted in polymeric (polypropylene) specimens. Interesting results have been obtained: a sure (from a quantitative and qualitative point of view) link between fracture toughness of the polymeric materials at hand and the experimental evidence of thermal phenomena around the cracks have been identified with repeatibility [1]. These phenomena had been theoretically anticipated [2], but literature-reported experiments treated only metallic specimens with partial crack growth.

The observed phenomenon (heating of the specimen broken ends in the absence of massive plastic flow) suggested the idea to determine the fracture energy by means of its thermal effects (the heating itself!) experimentally evaluated. In that research, IR thermography has been used as non-intrusive way to analyse the temperature variation fields in polymeric specimens. The analogic signal of IR camera is digitalized to obtain selective and quantitatively significant results.

The three types of polypropylene under study were supplied by Himont SpA (Ferrara, Italy) and a conventional mechanical analysis was performed on them. The related results and literature data are compared with thermomechanical analysis obtaining a substancial matching.

\section{The materials}

The specimens (after UNI 5819-66) of the three materials under study have been supplied directly by Himont SpA (Ferrara, Italy), courtesy of Dr POLATO. The cross section in the gauge zone is $1 / 2^{\prime \prime} \cdot 1 / 8^{\prime \prime}(12.7 \cdot 3.25 \mathrm{~mm}$. about). 
The materials are technical polypropylenes, hereinafter called: PP (omopolymer of polypropylene), PPE/E (eterofasic copolymer of propylene and ethylene) and PPE/R ("random" copolymer of propylene and ethylene) physical characteristics, as declared by the manufacturer, are: density $\mathrm{d}=902 \mathrm{~kg} / \mathrm{m}^{3}$; specific heat at constant pressure (at $293 \mathrm{~K}$ ) $\mathrm{c}_{\mathrm{p}}=2200 \mathrm{~J} / \mathrm{kg} \cdot \mathrm{K}$.

The relevant mechanical characteristics have been determined by means of the testing device Instron 8032 of DIEM-University of Bologna. RT tensile tests were performed at various strain rates, to detect the influence of time scale on the materials. The strain rates were scaled as follows:

$$
V 1: 0.0036 \mathrm{~s}^{-1} ; \quad V 2: 0.0114 \mathrm{~s}^{-1} ; \quad V 3: 0.036 \mathrm{~s}^{-1}
$$

The experiment campaign evidenced some common material behaviours at a specific deformation rate:

- V1: each material showed necking with oriented crystallization and subsequent plastic flow in the central zone, but no break was obtained within the device stroke (150 $\mathrm{mm}$, with a specimen gauge length of $100 \mathrm{~mm}$.);

- V2: the PP and PPE/E specimens broke after necking with oriented crystallization and plastic flow, the PPE/R behaved as for $V 1$;

- V3: each specimen (of each material) broke, exhibiting a more brittle behaviour with respect to the previous case (reduced plastic flow).

In table / the more significant results are reported (MPa).

\begin{tabular}{|l|c|c|c|}
\hline \multicolumn{4}{|l|}{ TABLE I - Experimental results obtained for different deformation rates } \\
\hline Material V2(V3) & PPE/E & PPE/R & PP \\
\hline$\sigma_{\text {MAX }(\mathrm{MPa})}$ & $29.7(31.7)$ & $--(33.3)$ & $38.4(39.7)$ \\
\hline Elongation on gauge lenght $100 \mathrm{~mm}(\mathrm{~mm})$ & $58.5(33.5)$ & $--(36.5)$ & $33.0(18.5)$ \\
\hline $\mathrm{E}$ (tangent in the origin) & $1000(1050)$ & $---(1190)$ & $1540(1600)$ \\
\hline
\end{tabular}

The reference fracture mechanics characteristics were obtained from:

a) literature data [3] related to direct determination of the fracture energy by means of the essential work of fracture method [4], which may be affected by experimental troubles and is rather conventional, because the influence of time scale is actually not taken into account;

b) Linear Elastic Fracture Mechanic approach via $K_{I C}$ and subsequent transformation to $J_{C}$, which is intrinsecally inaccurate from a theoretical point of view because of the viscoelastic nature of the material and of the non-linearity of the load-deformation relation.

Indeed, the situation from a scientific point of view is not completely satisfying. Previous studies [1] have accerted values with the same order of magnitude (the thermomechanical parameter being two or three times greater than the mechanical LEFM one), which is reputed insufficient to assess the quantitative validity of the proposed thermomechanical approach.

According to the results reported in [3], PP has a value of Essential Work Fracture of about $23 \mathrm{~kJ} / \mathrm{m}^{2}$ in Double Edge Notched Test specimens under 0.00014-0.00042 $\mathrm{s}^{-1}$ strain rates. The total fracture work for $6-6.5 \mathrm{~mm}$ specimen ligament (corresponding to $3 \mathrm{~mm}$. bilateral crack specimens of figure1, i.e. Bilateral Crack specimens) is about 70 $\mathrm{kJ} / \mathrm{m}^{2}$. 


\section{http://dx.doi.org/10.21611/qirt.1992.026}

The Authors have performed dead-loading fracture tests on $\mathrm{BC}$ specimens. The three $\mathrm{mm}$. cracks were inserted in the central zone (figure 1). Experimental data have been processed by the classical equations which allow to calculate the value of $K$, from the average stress and crack geometry and, eventually, the value of $J \cdot K / 2 / E$ for the case of plane stress. As above reported this way is not rigorous because the theory of stress-intensity factor $K$ is based upon the hypothesis of a perfectly linearelastic material. For high loading rates one may be obtain a behaviour quasi-elastic, i.e. the reduced dissipative component could be transformed into a deviation from linearity. This transformation should guarantee the objectivity of the fracture resistance hierarchy between analysed materials. The second simplification is the linearization of the load-deformation curve (figure 2) which identifies the value of $E$.

In table /I the results of such calculations are reported. SL means monotonic loading up to fracture, while DL means loading, unloading, monotonic loading up to fracture. The loading rate was $100 \mathrm{~N} / \mathrm{s}$, up to maximum loads smaller than $1 \mathrm{kN}$. Fractures, to be accepted, should occur within $30 \mathrm{~s}$ from loading beginning. The first load of $\mathrm{DL}$ tests was about $90 \%$ of the second load. For $E$, the values of secant moduli found during controlled strain rate tests have been used: $850 \mathrm{MPa}$ for PP, $560 \mathrm{MPa}$ for $\mathrm{PPE} / \mathrm{R}$ and $415 \mathrm{MPa}$ for PPE/E.

\begin{tabular}{|l|c|c|c|}
\hline \multicolumn{4}{|c|}{ TABLE II-Average energy per unit area of the fractured ligament } \\
\hline Material & PPE/E & PPE/R & PP \\
\hline $\mathrm{J}_{\mathrm{AV}}\left(\mathrm{kJ} / \mathrm{m}^{2}\right): \mathrm{SL}$ & 9.65 & 9.9 & 8.1 \\
\hline $\mathrm{DL}$ & 9.65 & 7.5 & 8.1 \\
\hline
\end{tabular}

\section{Thermomechanical analysis}

The thermomechanical analyses have been performed by means of the IR cameradevice described in paragraph 1. During the experiments of fracture resistance of paragraph 2. the thermal story of the specimen has been recorded and the phenomena of massive heating during fracture have been detected with high repeatibility on most specimens. In particular, hot spots (with temperature increase up to $5 \mathrm{~K}$ for PPE/E and to $1.5 \mathrm{~K}$ for the others) appeared in the fracture zones, even in the absence of evident plastic flows (figures $A^{*}$ and $B$ ). Indeed, in the fractured ends the materials (particularly PPE/E; in the other two materials the phenomenon is rather limited) exhibit well known [4] [5] physical modifications: elliptic zones (in the plane of the specimen) with an opaque white aspect instead of the translucent white one typical of the virgin material. These zones have the major axis coinciding with the fracture and spring from the tips of the cracks just before the sudden fracture, without significant deformations.

The working hypothesis is that the hot spots are the thermal effect of most part of fracture energy [1] which is trapped in the neighborhood of the crack because of the very low heat transmission coefficients involved, and that elliptic (oriented crystallization) zones belong to the store of dissipative physical modification strictly related to fracture propagation. A parameter $J_{A V}$ to measure the average energy per unit area of the fractured ligament (net section of the specimen) has been introduced

$$
J_{A V}=\frac{d \cdot C_{p} \cdot \int_{S}\left(T-T_{0}\right) d S}{I_{F}}
$$

\footnotetext{
* The colour plates of this article 26 are located on oage $I X$ of the colour gathering, at the end of the book
} 
were $I_{F}$ is the length of the ligament $(12.7 \mathrm{~mm}$ minus crack length) and $S$ is the surface of the specimen where heating appears, as showed by thermographies (that is in the plane of the specimen, which is inherently bidimensional). The evaluation of $J_{A V}$ is based upon the assumption of:

a) constant temperature variation $\left(T-T_{0}\right)$ along the thickness of the specimen;

b) no heat loss, which leads to underestimate the heating.

In table III the results obtained from several BC specimens (two or three experiments per datum) with $3 \mathrm{~mm}$ cracks are reported. The presumed accuracy is about $\pm 5 \%$ for PPE/E and $\pm 10 \%$ for the others. When the temperature variations were spread all over the specimen or smaller than $0.2 \mathrm{~K}$, the experiment has been rejected. Most PPE/R specimens showed such a behaviour and even the few succesful related experiments exhibit very low temperature variations.

TABLE III-Average energy per unit area of the fractured ligament for BC specimens

\begin{tabular}{|c|c|c|c|}
\hline Material & PPE/E & PPE/R & $P P$ \\
\hline $\mathrm{J}_{\mathrm{AV}}\left(\mathrm{kJ} / \mathrm{m}^{2}\right): \mathrm{SL}$ & 50. & $\ldots$ & 24. \\
\hline $\mathrm{DL}$ & 44. & 13. & 22. \\
\hline
\end{tabular}

In the case of some $3 \mathrm{~mm}$ monolateral cracks it was obtained: $67 \mathrm{~kJ} / \mathrm{m}^{2}$ for $\mathrm{SL}$ in $P P E / E$ and $39 \mathrm{~kJ} / \mathrm{m}^{2}$ for $\mathrm{DL}$ in PP.

\section{Conclusions}

Comparing table II, table III and fracture work values (after [3]) it is evident that thermomechanical analyses give intermediate values of fracture energy. This behaviour was expected on the basis of the considerations reported in paragraph 2 and of the following ones:

- Linear Elastic Fracture Mechanics, even resorting to a tricky choice of elastic modulus (secant modulus instead of Young modulus), cannot take into account the non-linearity of the phenomenon (see figure 2); accordingly, the fracture toughness is underestimated. Moreover, the dissipations around the tip are sensible.

- The Essential Work Fracture method [3] [4] is based upon constant strain rate fracture tests, lasting several tens of seconds. In this way the viscoelastic dissipations of the whole specimen may be of the same order of magnitude of the external work, an they sum up to actual local fracture work! As a consequence, this method leads to overestimate the total (specific) fracture work.

Every discussion about the separation of total (specific) work of fracture into EWF and nonessential work (the former related to the fracture surfaces, the latter related to plastic and crystallized zones) is behind the aim of this paper. Nevertheless, the few available data have been reported in the form of $J_{A V}$ versus ligament length diagram (figure 3). The trends for PP and PPE/E appear to be similar to those reported in [3] and [4].

It is the authors' opinion that the proposed thermomechanical method can be used to establish reliable toughness hierarchy between polymers. The validity of quantitative thermomechanical analyses via IR thermography is not completely assured (even if the same holds for the other analysis methods!). Further experiments are in progress on this way.

\section{Acknowledgements}

This work has been realized within a research programme subsidized by C.N.R. (Consiglio Nazionale Ricerche) Italy. 


\section{REFERENCES}

[1] MEDRI (G.) and RICCI (R.). - Thermomechanical analysis of cracked polymeric specimens. Plastic Rubber Proc, Appl. J. 15, 1, 1991.

[2] BUI (H.D.) et alii. - Thermomechanical coupling in fracture mechanics. In "Thermomechanical Couplings in Solids" (Bui H.D. and Nguyen Q.S. Eds), Elsevier Science Pub. (North-Holland), Amsterdam, 1987.

[3] LEVITA (G.) et alii. - La valutazione della resistenza alla frattura dei polimeri duttili mediante il metodo del lavoro essenziale di frattura (EWF) (The evaluation of fracture toughness of ductile polymers via essential work method). Proc. X Conv. Naz. AlAS, Pisa, 1992.

[4] MAI (Y.) and COTTEREL (B.) - On the essential work of ductile fracture in polymers. Int. J. Fracture, 32, 1986, p.105-125.

[5] BARTENEV (G.M.) and ZUYEV (Yu.S.). - Strength and failure of viscoelastic materials. Pergamon Press Ltd., Oxford, 1968.

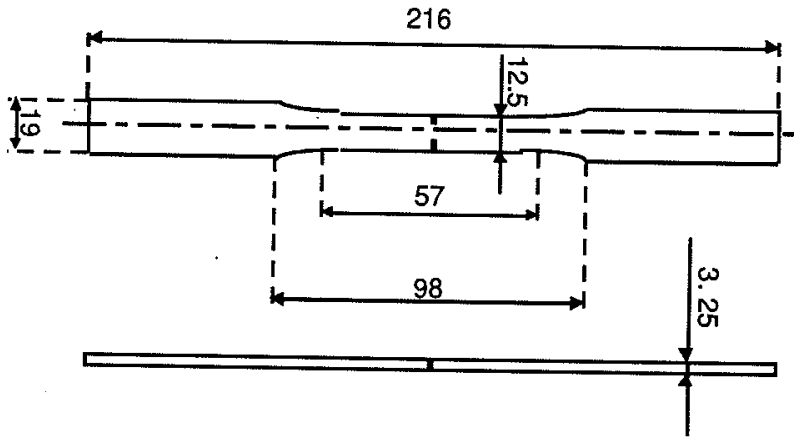

Fig. 1 . Standard test piece ( UNI 5819-66 )

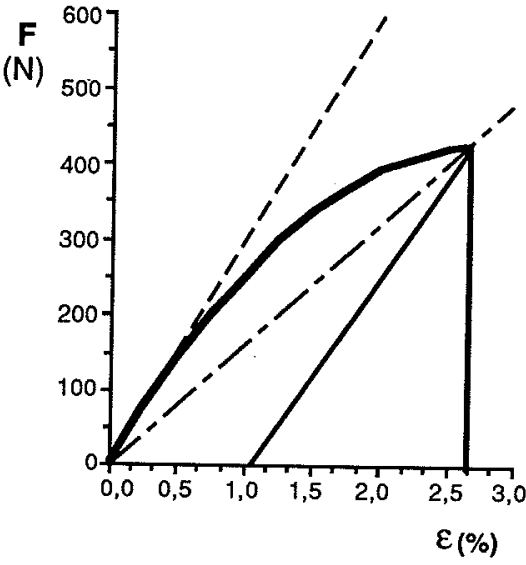

Fig. 2 . Load deformation curve for PP specimens with $6 \mathrm{~mm}$.monolateral crack at $\varepsilon=0.001 / \mathrm{s}$

- $-ー-$. Tangent Stiffness

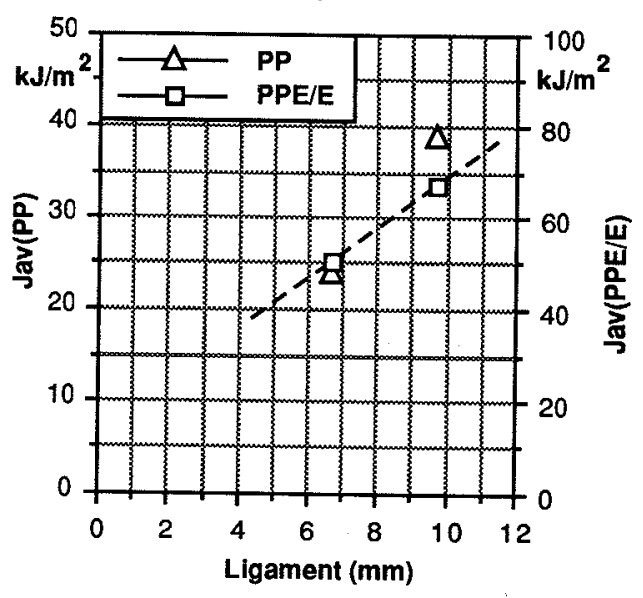

Fig. 3 . Jav versus ligament length 